Case Report

\title{
On Mathematical Modelling by EEE, ECE, ME Civil Post Graduate Students a Case Study Report
}

\author{
Johnwilliam Jebaraj \\ Department of Mathematics, Vivekanandha College of Engineering for Women, Trichengode (Tk), Namakkal (Dt), Tamil Nadu, India \\ Email address: \\ jebarajmal@gmail.com \\ To cite this article: \\ Johnwilliam Jebaraj. On Mathematical Modelling by EEE, ECE, ME Civil Post Graduate Students A Case Study Report. Teacher Education \\ and Curriculum Studies. Vol. 5, No. 2, 2020, pp. 42-45. doi: 10.11648/j.tecs.20200502.15
}

Received: January 8, 2020; Accepted: May 27, 2020; Published: June 8, 2020

\begin{abstract}
Recently the engineering institutes in India and abroad ignored the importance of mathematical modeling techniques in engineering teaching process and gave no places in their curriculum. In the present study the investigator applied random sampling on 15 post graduate engineering students, formulated four hypotheses connecting the innovative (attitude, relative advantage) and implementation variablion (utilisatation, satisfaction) and examined the relationships between the two variables. By using regression analysis, the result demonstrated that the two variables were significantly related. This implies the implementation of mathematical modeling in the engineering discipline was not successful. The investigator tried to identify the factors that would determine the successful implementation of mathematical modeling in the engineering discipline. It is critically important that mathematically trained and technologically competent research experts should be appointed and utilized as resources in the engineering research making bodies. Engineering research institutes with mathematical modeling facility should be collaborated with those that lack them to provide all research activities and opportunity to witness, learn from successful modeling related experiments.
\end{abstract}

Keywords: Post Graduate Engineering Students, Mathematical Modeling, Attitude, Relative Advantage, Utilization, Satisfaction, Scaffe's Post Hoc Test

\section{Introduction}

Recently the Engineering institutes in India and worldwide ignored the importance of mathematical modeling practices among the post engineering students and gave no place in their research curriculum. They ignored the critical input of mathematical modeling knowledge which is ultimately the key agents as regards conceptualizing and planning to make breakthrough. While ideally this radical departure from linear approach should have had a negative impact on post graduate engineering practices and technical viability of post graduate engineering teaching process, it nevertheless enjoyed significant success.

The application of mathematical modeling techniques become major problem in today's pharmaceutical research field. The investigator concerned with lack of mathematical modeling techniques and tried to identify the factors in order to improve the application of mathematics practices.
The immediate commitment of any engineering research organization is that all the research scholars must develop, sharpen and deepen their understanding mathematical modeling technique and its process. For this to occur a rigorous mathematical research curriculum must be recognized, taught and assessed in a problem solving environment

The post graduate engineering system in India viewed as driving force in development of our county [17]. Mathematical modeling may have an impact on major industrial problem such as cost reduction and improvement of the device performance and efficiency [8]. Creation of knowledge based technological innovation and their commercialization through technology incubation would boot scholar's participation in knowledge creation and transfer process [15]. By quickly building human resources with key knowledge and skill, the research organization can help to develop responsiveness in the educational market [18]. Studies revealed that research organization culture and 
research practices play an important role in any research process. The main issues, which emerged, have been presented here in the form of following questions.

Does the post graduate engineering student's attitude is directly related to satisfaction with mathematical modeling technology?

Does the post graduate engineering student's attitude is directly related to utilization of mathematical modeling technology?

Does the post graduate engineering student's relative advantage is directly related to satisfaction with mathematical modeling?

Does the post graduate engineering student's relative advantage is directly related to utilizing of mathematical modeling?

\section{Materials and Method}

\subsection{Sampling and Data Collection}

Samples of 15 post graduate engineering students (5-EEE, 5-ECE, 5-ME Civil), belong to Vivekananda College of Engineering for Women, Trichengodu, Namakkal (Dt), Tamil Nadu, INDIA were selected by random sampling for the study. Data was collected using a research tool developed by Rao (1993), which is specially prepared to measure attitude and innovative characteristics towards utilization and implementation of innovative process.

\subsection{Method of Analyses}

Spearman's correlation coefficient as well as by Scaffee's Post hoc test were used to chick relationships between innovative and implement variables.

\subsection{Result}

Majority of the respondents were unknown about mathematical modeling techniques. Among them only 55 percentage combined their attitude to 'aid educative instruction', 'to access engineering information', or 'to aid engineering administration'. The majority are in need of training in mathematical modeling technique. Among the engineering students majority study only few modeling journal. Percentage of members in any mathematical modeling association or user group is poor.

\subsubsection{Attitude and Relative Advantage}

The attitude of post graduate engineering students was generally positive towards mathematical modeling techniques. They believe mathematical modeling is a potential research tool, can bridge the educational gap and bring about better way of life for average research person. The data showed that the post graduate engineering students need to be assured that mathematical modeling techniques do not pose a threat to their research skills. Post graduate engineering students had a positive perception of the relative advantage compared to traditional method of research instruction. Mathematical techniques could enable quicker access to research administrative skills.

\subsubsection{Satisfaction and Utilization}

The post graduate engineering students perceived mathematical modeling as reliable, accurate, and meeting their innovative skills and they declared it as an essential research tool. They unable to ascertain the questions concerned with complexity. Some said mathematical modeling techniques did not enhanced their job as research scholars.

\section{Analyses on Hypotheses}

Table 1 shows that the Spearman's correlation coefficient for attitude, relative advantages, with utilization are 0.428 , 0.432 respectively. It is significant at 0.05 . It is found that there is a positive significant relationship between utilization with attitude, relative advantages.

Table 1. Relationship between utilization with attitude, relative advantages.

\begin{tabular}{llll}
\hline Dependent Variable & Independent Variables & Correlation Value (r) & Significant value \\
\hline \multirow{2}{*}{ Utilization } & Attitude & 0.428 & $\mathrm{P}>0.05$ \\
& Relative advantages & 0.432 & $\mathrm{P}>0.05$ \\
\hline
\end{tabular}

\subsection{Relationship Between Satisfaction with Variables Attitude, Relative Advantages}

Table 2 shows that the spearman's correlation coefficient for attitude, relative advantages, with satisfaction are $0.496,0.223$ respectively. It is significant at 0.05 . It is found that there is a positive significant relationship between satisfactions with attitude, relative advantages.

Table 2. Relationship between satisfaction with variables attitude, relative advantages.

\begin{tabular}{llll}
\hline Dependent Variable & Independent Variables & Correlation Value (r) & Significant value \\
\hline \multirow{2}{*}{ Satisfaction } & Attitude & 0.496 & $\mathrm{P}>0.05$ \\
& Relative advantages & 0.323 & $\mathrm{P}>0.05$ \\
\hline
\end{tabular}

\subsection{SCAFFEE'S Post Hoc Test for Utilization of Different Engineering Students}

Scaffee's Post hoc test is used to find out where the difference occurs between utilization of different engineering students. An attempt has been made to analyze it. 
Table 3. Post hoc test for satisfaction of different engineering students.

\begin{tabular}{llll}
\hline EEE & ECE & ME Civil & I-J \\
\hline 14.216 & 12.089 & & 2.127 \\
14.216 & & 11.237 & 2.979 \\
& 12.089 & 11.237 & 0.852 \\
\hline
\end{tabular}

The Scaffee's post hoc test shows that utilization of mathematical modeling for EEE students are higher than the students of ECE and BE Civil, Utilization of mathematical modeling for ECE are higher than the BE Civil students.

\subsection{SCAFFEE'S Post Hoc Test for Satisfaction of Different Engineering Students}

Scaffee's Post hoc test is used to find out where the difference occurs between satisfactions of different engineering students. An attempt has been made to analyze it.

Table 4. Post hoc test for satisfaction of different engineering students.

\begin{tabular}{llll}
\hline EEE & ECE & ME. Civil & I-J \\
\hline 45.276 & 36.265 & 32.117 & 6.511 \\
40.876 & & 32.118 & 8.759 \\
& 34.265 & 1.048 \\
\hline
\end{tabular}

The Scaffe's post hoc test shows that satisfaction of mathematical modeling for EEE students are higher than the students of ECE and BE Civil, satisfaction of mathematical modeling for ECE students are higher than the BE Civil students.

\section{Graphical Representation of Mean Score Difference Between Respondents}

Figure 1 shows the mean score difference between EEE, ECE, and ME Civil students and utilization, satisfaction of mathematical modeling. It reveals that satisfaction of mathematical modeling among EEE, ECE and BE Civil students is greater than utilization of mathematical modeling.

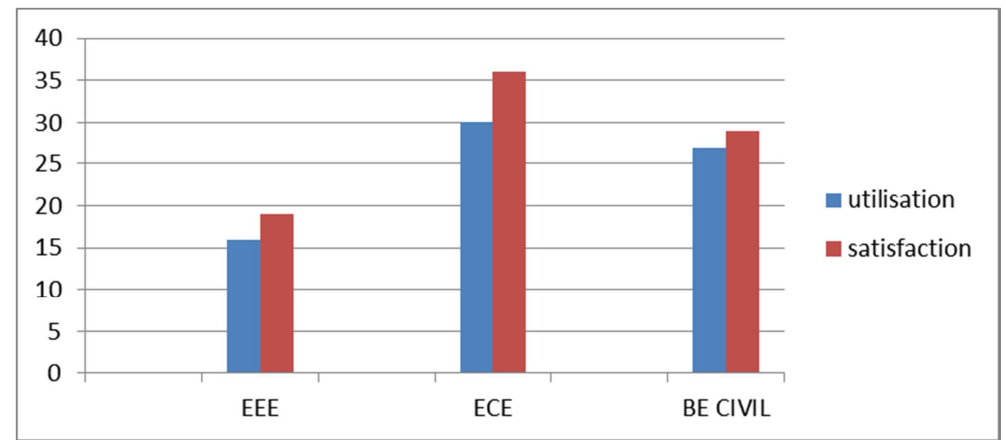

Figure 1. Graphical Representation of mean score difference between EEE, ECE, and ME Civil students and utilization, satisfaction of mathematical modeling.

\section{Discussion}

The present investigation study is aimed at improving practice in the post graduate engineering students by implementing mathematical modeling techniques have the following research implications The importance of attitude, relative advantage in the utilization and satisfaction process underscores the need for strategic planning in mathematical modeling in EEE, ECE BE engineering teaching and training, designed to accomplish specific curriculum objectives.

The literature notes that making modeling compatible with ECE and BE Civil student's tasks will boost its utilization and satisfaction by specifying its role more precisely. mathematical modeling practice should be configured to accomplish specific tasks such as drawing conclusion, creative, innovative, logical thinking, abstract thinking, drawing inference, inter connections etc. EEE, ECE and BE Civil department curriculum should make guidelines for the effective use of modeling technology. The engineering educators must understand the direction of innovative scientific progress and can update their mathematical modeling technology training programs to meet emerging industrial need.

Strong commitment from post EEE, ECE and BE Civil students to embrace mathematical modeling technology is essential in attaining the country's goals of mathematical modeling use. Active involvement of ECE and BE Civil s students who are users and non-users is a practical means of moving the mathematical modeling initiative forward cadre of post graduate engineering students motivated by potential of mathematical modeling techniques can ignite the interest of others become users. 


\section{Conclusion}

Consequently, the goal of this study was to identify this factors which leads to successful implementation of mathematical modeling in the EEE, ECE and ME Civils programme. Finally the shared involvement and leadership quality of pharmaceutical research scholars as well as policy makers facilitate a 'buy in' element that will guarantee the successful implementation of mathematical modeling technology throughout engineering education in India and in research community at large

\section{Acknowledgements}

The authors wish to thank the management for giving permission to do a case study, editor and the anonymous reviewers for their constructive critique that helped for the improvement of this paper.

\section{References}

[1] Cassady, J. (1998). Students and Instructor perceptions of the efficacy of computer aided lectures in undergraduate university course. Journal of Educational Computing Research, 19 (2), 175-189.

[2] Dolk, D. R. (1988). Model management and structured modeling: The role of information Resources Dictionary System. Comm. ACM, 31: 6, 704-718.

[3] Dolk, D., Kothemann, J. (1993). Model integration and the theory of Models. Decision Support System, 9 (1), 51-63.

[4] Fishbein, M., \& Ajen, I. (1975). Belief, Attitude, Intension and Behaviors: An Introduction to theory and Mathematical modeling in engineering, MA: Addison-Wesley.

[5] Flick, L., \& Bell, R. (2000). Preparing tomorrow science teachers to use Technology. Guide Lines for Science educators Contemporary issues in technology and teacher education 1 (1), 39-60.

[6] Garry Willgoose, S. (2005). Mathematical modeling of whole landscape evolution. Earth and Bio sphere Institute. School of Geography, University of Leeds, Leeds, UK.

[7] Gratian-Guinness. (1997). History of Mathematical Sciences, London.
[8] Jean Piere, H., \& Boeuf, A. (2004). The design and implementation of a prototype structures modeling environment. Annals of operations research, 38, 453-484.

[9] John. Jebaraj. (2011). Impact of Mathematical modeling on Modern Sciences. Asian Journal of Microbiology, Biotechnology and Management Sciences, 13 (3), 489-494.

[10] John. Jebaraj. (2011). Evaluating mathematical modeling in the biological research set up. Asian Journal of Microbiology, Biotechnology and Environmental Sciences, 13 (3), 515-518.

[11] Khaparde, M. S. (1998). Action research Process. National Council of Educational Research and Training, New Delhi.

[12] Leaderman, N., \&Niess, M. (2000). Technology for technology's sake or for the improve teaching and learning? School science and Mathematics, 100 (7), 345-348.

[13] Mathematical Drawing and Modeling. (1994). Encyclopedia Britannica: A Dictionary of Arts, Science and General Literature, 9th Edition, 628-629.

[14] National Science Teachers Association. (1990). Science Teachers speak out the NASTA lead paper on Science and Technology Education for 21st century, Washington, Dc: Author.

[15] Parimal Mandke, F. (2007). Promoting Culture of Research in Universities. Universities News, 4 (2), 4-7.

[16] Ponnusamy, A., \&Antti Rasila, D. (2007). Planner Harmonic Mappings. Mathematics letter, Ramanujanm Mathematical Society 17 (2), 56-65.

[17] Rogers, E. M. (1983). Diffusion of Innovations. New York: Free press.

[18] Sachi Hatakenaka, P. (2007). The Role of Higher Education Institutions in Innovation and Economic Development. New Frontiers in Education, 3 (4), 123-127.

[19] International Center for Numerical Methods in Engineering (2008). 5th European congress on computational mechanics. ECCOMAS $2008 . \quad$ Available http://congress.cimne.com/iacm08/frontal/Stats.asp/ (retrived July 4, 2008).

[20] National Science Teachers Association. (1999). N STA position Statement: The use of computer in Science education. Available http://www.nsta.org/ (retrived September 12, (1999). 